\title{
Cells of origin of squamous epithelium, dysplasia and cancer in the head and neck region after bone marrow transplantation
}

\author{
YOSHIHIRO KANO ${ }^{1,2}$, HIDESHI ISHII ${ }^{1}$, MASAMITSU KONNO ${ }^{1}$, MAKOTO YAMASAKI ${ }^{2}$, HIROSHI MIYATA ${ }^{2}$, \\ SHIMPEI NISHIKAWA ${ }^{1,2}$, ATSUSHI HAMABE ${ }^{1,2}$, HISATAKA OGAWA ${ }^{1,2}$, HIDEKAZU TAKAHASHI ${ }^{2,3}$, \\ KATSUYA OHTA $^{1,2}$, SHINICHIRO HASEGAWA ${ }^{1,2}$, KOUJI TANAKA ${ }^{2}$, TAKAHITO FUKUSUMI ${ }^{1,4}$, \\ MASAHISA OTSUKA $^{2}$, KOICHI KAWAMOTO ${ }^{2}$, NAOTSUGU HARAGUCHI ${ }^{2}$, RIKA FUJIMOTO ${ }^{5}$, \\ MASAHARU ISOBE ${ }^{5}$, YASUHIKO TOMITA ${ }^{6}$, NARIAKI MATSUURA ${ }^{7}$, \\ SHUJI TAKIGUCHI ${ }^{2}$, MASAKI MORI ${ }^{2}$ and YUICHIRO DOKI ${ }^{2}$ \\ Departments of ${ }^{1}$ Frontier Science for Cancer and Chemotherapy, ${ }^{2}$ Gastroenterological Surgery, Osaka University, \\ Graduate School of Medicine, Osaka, Japan; ${ }^{3}$ Dana-Farber Cancer Institute, Harvard Medical School, Boston, \\ MA 02215, USA; ${ }^{4}$ Department of Otorhinolaryngology-Head and Neck Surgery, Osaka University, \\ Graduate School of Medicine, Osaka; ${ }^{5}$ Laboratory of Molecular and Cellular Biology, Department of Materials \\ and Biosystem Engineering, Faculty of Engineering, Toyama University, Toyama; ${ }^{6}$ Department of Pathology, \\ Osaka Medical Center for Cancer and Cardiovascular Diseases, Osaka; ${ }^{7}$ Department of Functional \\ Diagnostic Science, Osaka University, Graduate School of Medicine, Osaka, Japan
}

Received July 15,2013; Accepted August 26, 2013

DOI: 10.3892/ijo.2013.2206

\begin{abstract}
Secondary solid tumors that occur after hematopoietic stem cell transplantation (HSCT) are late complications of HSCT. Previously, secondary solid tumors were considered to be recipient-derived cells because transplanted cells do not contain epithelial cells. Recently, however, not only donor-derived epithelial cells but also donor-derived secondary solid tumors have also been reported in mice and humans. It means that circulating bone marrow-derived stem cells (BMDCs) including hematopoietic stem cells include the stem cells of many tissue types and the precancerous cells of many solid tumors. In most reports of donor-derived secondary solid tumors, however, tumors contained a low proportion of BMDC-derived epithelial cells in mixed solid tumor tissues. To our knowledge, there are only five known cases of completely donor-derived tumor tissues, i.e., four oral SCCs and a pharyngeal SCC. In this study, we analyzed five human clinical samples of solid tumors, i.e., two esophageal squamous cell carcinomas (SCCs), two oral SCCs and a tongue carcinoma. In the oral and tongue, completely donor-derived tissues were not observed, but in esophagus a completely donor-derived esophageal epidermis and SCC were observed for the first time. In addition, in another esophageal SCC patient, a completely donor-derived dysplasia region of
\end{abstract}

Correspondence to: Professor Hideshi Ishii, Department of Frontier Science for Cancer and Chemotherapy, Osaka University Graduate School of Medicine, 2-2, Yamadaoka, Suita, Osaka 565-0871, Japan

E-mail: hishii@gesurg.med.osaka-u.ac.jp

Key words: cell of origin, FISH analysis, squamous cell carcinoma esophageal epidermis was observed near recipient-derived SCC. This study suggests that BMDC-derived cells include the stem cells of esophageal epidermis and the precancerous cells of esophageal SCC and can differentiate into esophageal epithelium and esophageal SCC.

\section{Introduction}

Recent studies on regenerative medicine have indicated that mesenchymal tissues contain multipotent stem or progenitor cells that can give rise to neural and skin tissues, adipocytes, myocytes, cardiomyocytes, blood vessels, chondrocytes and hepatocytes (1). Given that bone marrow (BM) and adipose tissues are major sources of mesenchymal stem or progenitor cells (2), the observation of BM transplantation in mice and humans can provide adequate biological and biophysical information concerning cells of origin. Reportedly, bone marrow-derived cells (BMDCs) accumulate in the gastric epithelium as a result of Helicobacter pylori infection and can contribute to tumor development, indicating that infection can lead to the development of hyperplasia, metaplasia and dysplasia associated with BMDC recruitment and accumulation in the gastric epithelial mucosa, which occurs against a background of chronic inflammation (3). However, the contribution of BMDSs to head and neck cancers, including esophageal cancer, remains unknown.

GVHD is a major complication of allogeneic hematopoietic stem cell transplantation (HSCT), with significant morbidity and mortality (4). Therefore, adequate control of GVHD is critical to the continued success of transplantation. GVHD shares its molecular basis with chronic inflammation. This molecular basis includes the induction of intrinsic damage 
Table I. Characteristics of cases with secondary SCCs after HSCT.

\begin{tabular}{|c|c|c|c|c|c|c|c|}
\hline \multirow[b]{2}{*}{$\begin{array}{l}\text { Case } \\
\text { no. }\end{array}$} & \multicolumn{4}{|c|}{ Blood diseases } & \multicolumn{3}{|c|}{ Secondary SCCs } \\
\hline & $\begin{array}{l}\text { Gender } \\
\text { of donor/ } \\
\text { recipient }\end{array}$ & Diagnosis & $\begin{array}{l}\text { Type of } \\
\text { HSCT }\end{array}$ & GVHD & Location & $\begin{array}{c}\text { Age at } \\
\text { diagnosis }\end{array}$ & $\begin{array}{l}\text { Time after } \\
\text { transplantation } \\
\quad(\text { months })\end{array}$ \\
\hline 1 & $\mathrm{~F} / \mathrm{M}$ & $\begin{array}{l}\text { Non-Hodgkin's } \\
\text { lymphoma }\end{array}$ & PBSCT & Chronic & Esophagus & 42 & 115 \\
\hline 2 & $\mathrm{~F} / \mathrm{M}$ & $\begin{array}{l}\text { Non-Hodgkin's } \\
\text { lymphoma }\end{array}$ & PBSCT & Chronic & Esophagus & 77 & 120 \\
\hline 3 & $\mathrm{~F} / \mathrm{M}$ & MDS & BMT & Chronic & Oral cavity & 38 & 76 \\
\hline $\begin{array}{l}4-1 \\
4-2\end{array}$ & $\mathrm{M} / \mathrm{F}$ & CML & $\mathrm{BMT}$ & Chronic & $\begin{array}{c}\text { Tongue } \\
\text { Oral cavity }\end{array}$ & $\begin{array}{l}45 \\
46\end{array}$ & $\begin{array}{l}150 \\
163\end{array}$ \\
\hline
\end{tabular}

F, female; M, male; MDS, myelodysplastic syndromes; CML, chronic myelogenous leukemia; HSCT, hematopoietic stem cell transplantation; PBSCT, peripheral blood stem cell transplantation; BMT, bone marrow transplantation.

to tissue stem or progenitor cells and deleterious effects on immune surveillance, all extensive and dynamic alterations that accumulate in the course of carcinogenesis (5). The use of immunosuppressant therapy exerts a favorable effect by ameliorating chronic GVHD, but it is associated with a higher relapse rate of hematopoietic and secondary malignancies, thus posing a major threat in the long term (6).

Cells for HSCT are obtained from BM, peripheral blood, or umbilical cord blood, which is proposed to contain mesenchymal stem or progenitor cells as well as other cell types $(7,8)$. Secondary malignancies following HSCT are common late complications (9). With regard to the cells of origin, secondary tumors are considered to be derived from recipient-derived cells because there are very few epithelial cells in normal BM and peripheral blood (10). Nevertheless, the contribution of human BM cells, including HSCs, to epithelium, dysplasia and cancer is poorly understood (11). To study the involvement of BM cells in solid tumors developing after HSCT and distinguish the origins of epithelial cancer cells in humans, we performed highly sensitive FISH using gender chromosome-specific probes and histopathological analyses in five cases of head and neck tumors that developed subsequent to gender-mismatched BM transplantation. Our study allowed the identification of donor-derived epithelium, dysplasia, and cancer of the esophagus against a background of chronic inflammation due to GVHD, demonstrating that BMDCs can contribute to the development of precancerous lesions and various cancers.

\section{Patients and methods}

Patients. Patient characteristics are summarized in Table I. All patients had received gender-mismatched HSCTs and developed GVHD. Two clinical samples were obtained from patients with esophageal squamous cell carcinoma (SCC) treated at our hospital. Three clinical samples from two patients with oral SCC and one patient with tongue Diseases (Osaka, Japan). All clinical samples used in this study were acquired after obtaining written informed consent from each patient.
FISH analysis. FISH analysis was performed by Chromosome Science Labo, Inc. (Sapporo, Japan) using formalin-fixed, paraffin-embedded tissue sections as described previously (12). Briefly, 5-mm-thick sections were deparaffinized, dehydrated, microwaved $(600 \mathrm{~W})$ in $2 \mathrm{X}$ saline sodium citrate (SSC) for $10 \mathrm{~min}$, cooled in PBS, digested in pepsin solution containing $0.1 \mathrm{~N} \mathrm{HClat} 37^{\circ} \mathrm{C}(0.1 \%$ pepsin for $10 \mathrm{~min}$ for samples $4-1$ and $4-2$; $0.02 \%$ pepsin for $5 \mathrm{~min}$ for the other samples), and dehydrated. Human XY FISH probes (Chromosome Science Labo, Inc.) were applied to the pretreated sections, covered with cover slips, and simultaneously denatured at $90^{\circ} \mathrm{C}$ for $13 \mathrm{~min}$. Hybridization was performed at $37^{\circ} \mathrm{C}$ overnight. Sections were then washed with $50 \%$ formamide/2X SSC at $37^{\circ} \mathrm{C}$ for $20 \mathrm{~min}$ and $1 \mathrm{X} \mathrm{SSC}$ for $15 \mathrm{~min}$ at room temperature. The slides were treated with antibodies at $37^{\circ} \mathrm{C}$ for $30 \mathrm{~min}$, washed three times with $0.1 \%$ Nonidet P-40/2X SSC, counterstained with 4',6-diamidino2-phenylindole (DAPI), and mounted. The FISH images were captured using the CW4000 FISH application program (Leica Microsystems Imaging Solution, Ltd., Cambridge, UK) using a cooled charge-coupled device camera mounted on a Leica DMRA2 microscope (Leica Microsystems, Wetzlar, Germany). The enumeration probes for the $\mathrm{X}$ chromosomes were labeled with cyanine 3 (Cy3), SpectrumGold ${ }^{\mathrm{TM}}$ (Abbott Laboratories, Abbott Park, IL, USA), or Cy5, whereas the enumeration probes for the Y chromosomes were labeled with SpectrumGreen ${ }^{\mathrm{TM}}$ or SpectrumRed ${ }^{\mathrm{TM}}$ (Abbott Laboratories). The number of cells showing FISH signals was counted by the observation of at least five fields under the microscope (x100). All data were evaluated by at least three pathologists.

Histopathological analyses. Pathological diagnoses of DAPI- or hematoxylin and eosin-stained samples were performed by at least three pathologists to identify normal and malignant cells.

\section{Results}

BM significantly contributes to normal epithelium and SCC of the esophagus. Esophageal cancer developed in a male recipient with non-Hodgkin's lymphoma 115 months after 

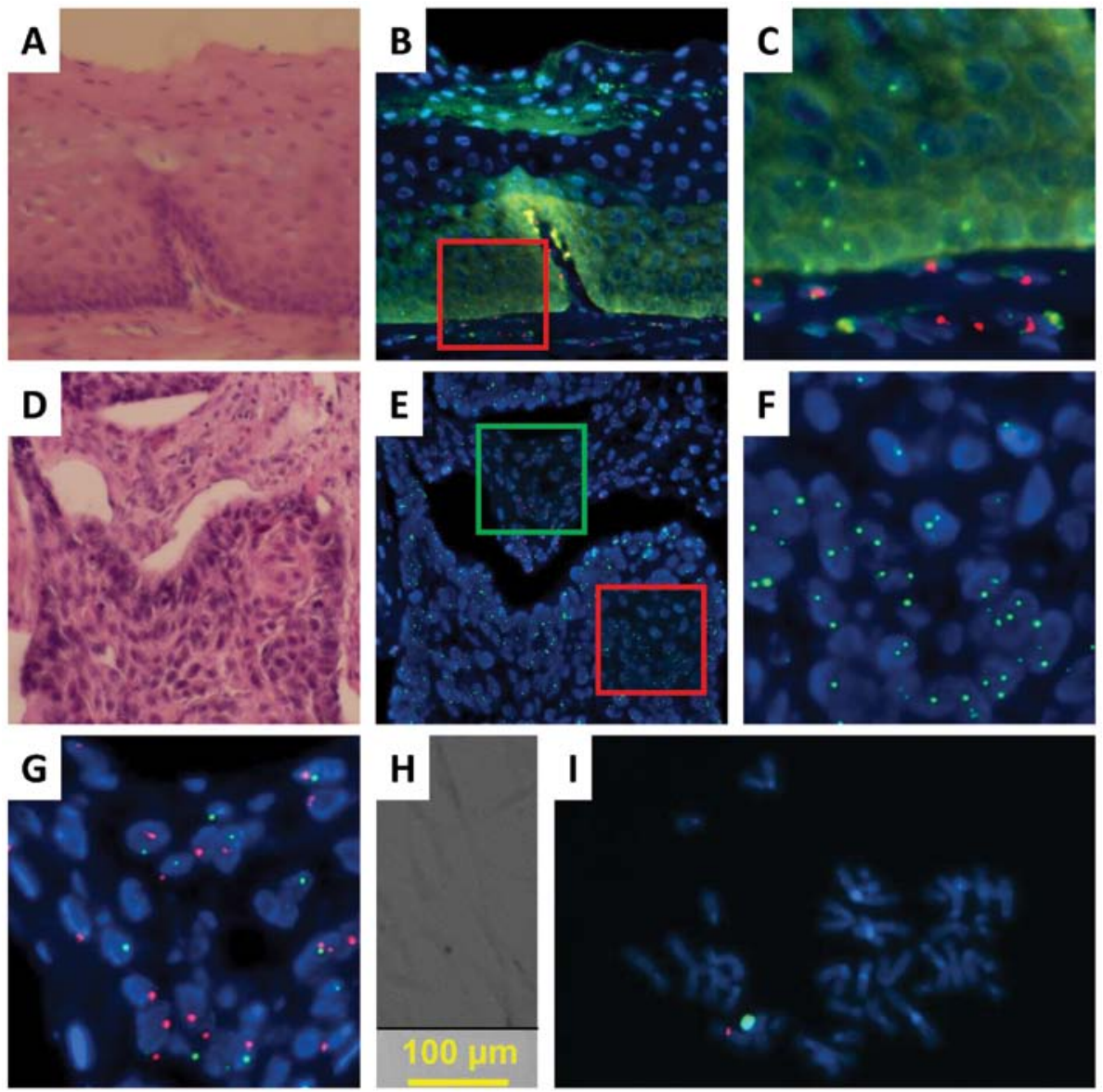

Figure 1. Donor-derived esophageal epithelial cells, SCCs, and recipient-derived fibroblasts from a male recipient (case 1) of peripheral blood stem cells from a female donor. (A) Tissue sections of the normal mucosa are stained with hematoxylin and eosin. (B) Neighboring tissue sections of (A) are examined by FISH to determine the centromeres of the X (Cy5, green) and Y (SpectrumRed, red) chromosomes. (C) A magnified image of the red-framed square in (B). (D) Tumor tissue sections are stained with hematoxylin and eosin. (E) Neighboring tissue sections of (D) are examined by FISH to determine the centromeres of the X (Cy5, green) and Y (SpectrumRed, red) chromosomes. (F) A magnified image of the cancerous region, i.e., the red-framed square in (E). (G) A magnified image of the stromal region, i.e., the green-framed square in (E). (H) Fibroblasts from the stromal region in a dish. (I) Fibroblasts from (H) are examined by FISH to determine the centromeres of the X (Cy3, red) and Y (SpectrumGreen, green) chromosomes. Panels A through F, x63 magnification; inset in panel D, x160 magnification.

gender-mismatched HSCT using cells from a female donor (Table I, case no. 1). To assess normal epithelium in the esophagus, FISH was performed using gender chromosome-specific probes (Cy5 and SpectrumGreen for the $\mathrm{X}$ chromosome; SpectrumRed for the $\mathrm{Y}$ chromosome; Fig. 1). A comparison of FISH and histopathological analyses indicated that all infiltrating lymphocytes in an examination of 50 fields displayed green $(\mathrm{X})$ signals but not red $(\mathrm{Y})$ signals, suggesting that the lymphocytes were replaced with donor hematopoietic cells after HSCT (representative data in Fig. 1A-C; Tables II and III). The data indicated that both epithelial cells and basal cells were also positive for green $(\mathrm{X})$ signals but not red $(\mathrm{Y})$ signals, whereas mesenchymal cells in the stroma were positive for both green $(\mathrm{X})$ and red $(\mathrm{Y})$ signals, suggesting that the recipient cells were integrated in the mesenchymal tissues (Fig. 1A-C). The infiltration of lymphocytes with only green (X) signals in the epithelial and mesenchymal tissues was compatible with chronic GHVD. The present data propose that epithelial regions within the esophagus were replaced predominantly with donor-derived cells after HSCT. Next, we examined tumor tissues obtained from case 1. Comparative FISH and histopathological analyses indicated that all SCC cells within the tumor displayed green $(\mathrm{X})$ signals but not red $(\mathrm{Y})$ signals, suggesting that esophageal cancer cells in case 1 were derived from donor cells in the regions examined. Infiltrating recipient-derived cells were not detected while lymphocytes with green (X) signals were present; this was compatible with the replacement of recipient hematopoietic cells by donor cells (Fig. 1D-G; Tables II and III). An examination of the mesenchymal tissues indicated that stromal cells, which include fibroblasts as well as lymphocytes and coexist in flanking regions of epithelial cancer cells, displayed red (Y) signals showing the contribution of the recipient cells. On the other hand, approximately $30 \%$ of the stromal cells displayed green (X) signals but not red signals, suggesting the involvement of donor-derived cells. We found that tumor-associated fibroblasts displayed both green (X) and red $(\mathrm{Y})$ signals, indicating that they were recipient cells (Fig. 1H and I).

Significant contribution of BM to dysplasia of esophagus. In case 2, the recipient was a male and the donor was a female. Esophageal cancer, which was characterized by dysplastic lesions, developed in the recipient 120 months after HSCT for malignant lymphoma, although the original disease 
Table II. Cell of origin.

\begin{tabular}{|c|c|c|c|c|c|c|c|c|}
\hline \multirow[b]{2}{*}{ Case no. } & \multicolumn{4}{|c|}{ Donor cells } & \multicolumn{4}{|c|}{ Recipient cells } \\
\hline & $\mathrm{E}$ & $\mathrm{L}$ & $\mathrm{D}$ & $\mathrm{C}$ & $\mathrm{E}$ & $\mathrm{L}$ & $\mathrm{D}$ & $\mathrm{C}$ \\
\hline 1 & + & + & ND & + & - & - & ND & - \\
\hline 2 & \pm & + & + & \pm & + & - & - & + \\
\hline 3 & \pm & + & ND & \pm & + & - & ND & + \\
\hline 4-1 & - & + & ND & - & + & - & ND & + \\
\hline $4-2$ & - & + & ND & - & + & - & ND & + \\
\hline
\end{tabular}

E, normal epithelial cells; L, peripheral mononuclear cells or lymphocytes; D, dysplasia; C, cancer cells; + , present; \pm , less than $5 \%$ involved; -, absent; ND, not detected. The data summarizes the results of Table III.

was in complete remission (Table I). As indicated in Fig. 2 (SpectrumGold for the X chromosome; SpectrumRed for the Y chromosome), FISH and histopathological analyses indicated that normal epithelial regions (lesion I in Fig. 2A) were composed predominantly of squamous cells as indicated by the yellow $(\mathrm{X})$ and red $(\mathrm{Y})$ signals, whereas a fraction displayed yellow $(\mathrm{X})$ signals alone, suggesting that the esophageal epithelium was reconstituted at least partially with donor-derived cells in the regions examined. As indicated by the representative data in Fig. 2B-D (Tables II and III), the nuclei were similar to those of the surrounding epithelial cells in size, indicating that they were also epithelial cells. Infiltrated lymphocytes displayed only yellow (X) signals, indicating that the hematopoietic cells in the recipient were replaced by donor cells and the involvement of GVHD. Histopathological analysis of the surgical specimen from case 2 indicated dysplasia (lesion II in Fig. 2A) as a continuous lesion with the tumor (lesion III). FISH and histopathological analyses of lesion II indicated that almost all the dysplastic cells displayed only yellow (X) signals but not red (Y) signals (Fig. 2E-G; Tables II and III), suggesting that the donor-derived cells were recruited to the esophageal epithelium and transformed or that some damaged donor cells may have been incorporated into the esophagus. Analysis of the tumor (lesion III; Fig. 2H-M; Tables II and III) showed both yellow (X) and red ( $\mathrm{Y}$ ) signals in almost all cancer cells, whereas a few cells expressed only yellow (X) signals (arrows in Fig. 2M; their nuclei were similar to those of the surrounding epithelial cancer cells in size), indicating that the tumor was composed predominantly of recipient cancer cells with a fraction of donor-derived cells. This was characterized by infiltrating lymphocytes displaying yellow (X) signals alone (arrows in Fig. 2J; the nuclei were much smaller than those of epithelial cells, indicating lymphocytes), thus being compatible with GVHD.

A mixed tumor of recipient- and donor-derived cells. In case 3 , the donor was a female and the recipient was a male who developed oral SCC 76 months after HSCT for myelodysplastic syndrome (Table I). FISH and histopathological analyses indicated that all infiltrating lymphocytes displayed green $(\mathrm{X})$ signals but not red $(\mathrm{Y})$ signals, suggesting that the lymphocytes were replaced after transplantation; this was again compatible with GVHD (Cy5 for the X chromosome; SpectrumRed for the Y chromosome; Fig. 3). Analysis of the normal epithelium indicated that almost all epithelial cells displayed red (Y) signals (Fig. 3A-C; Tables II and III), whereas approximately $5 \%$ cells displayed green $(\mathrm{X})$ signals alone. The nuclei of the latter cells were similar to those of the surrounding epithelial cells in size (Fig. 3C), indicating that normal epithelium in the surgical specimen of the oral cavity was composed predominantly of recipient cells. In the tumor, approximately $80 \%$ cancer cells expressed red (Y) signals (Fig. 3D-F; Tables II and III), whereas $20 \%$ cells displayed green $(\mathrm{X})$ signals alone. The nuclei of the latter cells were similar size to those of the surrounding SCC cells in size (Fig. 3F), suggesting that the tumor had two different origins: a predominant contribution from the recipient cells and a partial contribution from the donor cells, which may have developed after HSCT presumably through the involvement of GVHD.

Sequentially occurring oral cancer after HSCT. We encountered a patient who developed oral SCC on different tongue regions 150 (case 4-1) and 163 months (case 4-2) after single HSCT for chronic myeloid leukemia. The recipient was a female and the donor was a male. FISH and histopathological analyses showed that all infiltrating lymphocytes displayed red (Y) signals, indicating that these lymphocytes were replaced after

Table III. Results of this study.

\begin{tabular}{|c|c|c|c|c|c|c|c|c|c|c|c|c|}
\hline \multirow[b]{2}{*}{ Case no. } & \multicolumn{4}{|c|}{ Normal epithelial cells } & \multicolumn{4}{|c|}{ Cancer cells } & \multicolumn{4}{|c|}{ Dysplasia } \\
\hline & $\mathrm{Y}$ & $\mathrm{X}$ & $X Y$ & $\mathrm{XX}$ & $\mathrm{Y}$ & $X$ & $X Y$ & $X X$ & $\mathrm{Y}$ & $X$ & $X Y$ & XX \\
\hline 1 & 0.0 & 94.3 & 0.0 & 5.7 & 0.0 & 58.2 & 0.0 & 41.8 & ND & ND & ND & ND \\
\hline 2 & 36.1 & 18.1 & 41.0 & 1.2 & 27.9 & 34.9 & 65.1 & 2.3 & 0.0 & 86.7 & 0.0 & 13.3 \\
\hline 3 & 43.8 & 30.8 & 24.6 & 0.3 & 35.9 & 37.5 & 18.8 & 1.6 & ND & ND & ND & ND \\
\hline $4-1$ & 0.0 & 57.7 & 0.0 & 28.2 & 0.0 & 44.6 & 0.0 & 46.4 & ND & ND & ND & ND \\
\hline $4-2$ & 0.0 & 18.9 & 0.0 & 66.2 & 0.0 & 50.9 & 0.0 & 25.5 & ND & ND & ND & ND \\
\hline
\end{tabular}

Y, Y chromosome; X, X chromosome; XY, X and Y chromosomes; XX, two X chromosomes; ND, not detected. Scores in this table display percentages of each cell to all cells with signals. See Materials and methods. The data summarized in Table II. 


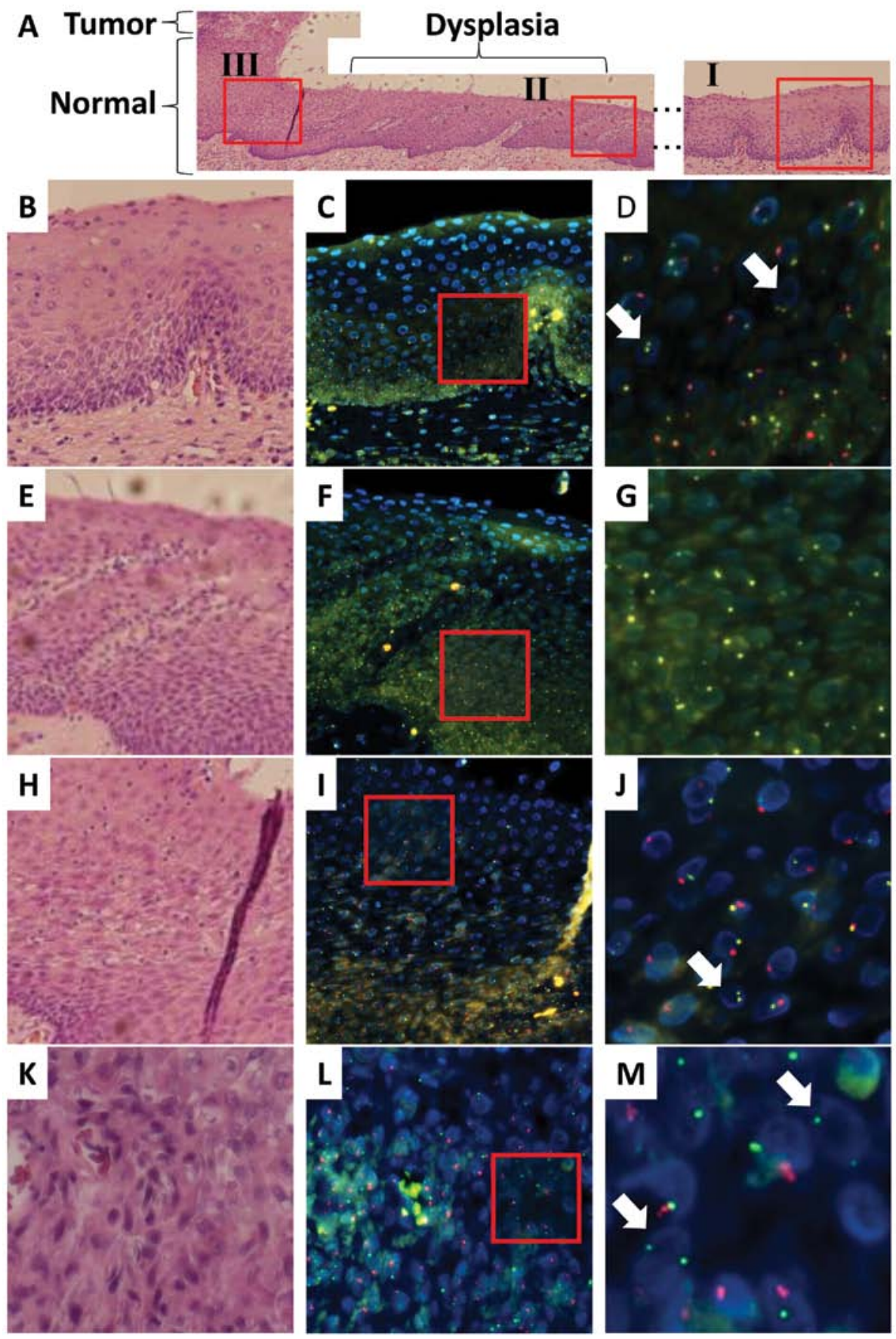

Figure 2. Recipient- and donor-derived esophageal epithelial cells and recipient-derived esophageal SCC from a male recipient (case 2) of peripheral blood stem cells from a female donor. (A) Tissue sections of the normal mucosa and solid tumor tissue are stained with hematoxylin and eosin. (B) A magnified image of normal mucosa (I). (C) Neighboring tissue sections of (B) are examined by FISH to determine the centromeres of the X (SpectrumGold, yellow) and Y (SpectrumRed, red) chromosomes. (D) A magnified image of the red-framed square in (C). A few donor-derived cells display XX signals, and their nuclei are similar to those of normal epithelial cells in size (white arrow). (E) A magnified image of the region with dysplasia (II). (F) Neighboring tissue sections of (E) are examined by FISH to determine the centromeres of the X (SpectrumGold, yellow) and Y (SpectrumRed, red) chromosomes. (G) A magnified image of the red-framed square in (F). No cell in the epithelium displays Y signals. (H) A magnified image of normal mucosa (III). (I) Neighboring tissue sections of (H) are examined by FISH to determine the centromeres of the X (SpectrumGold, yellow) and Y (SpectrumRed, red) chromosomes. (J) A magnified image of the redframed square in $(\mathrm{H})$. Infiltrating donor-derived cells display XX signals and smaller nuclei than those of normal epithelial cells (white arrow). (K) Tumor tissue sections are stained with hematoxylin and eosin. (L) Neighboring tissue sections of $(\mathrm{K})$ are examined by FISH to determine the centromeres of the X (Cy5, green) and Y (SpectrumRed, red) chromosomes. (M) A magnified image of the cancer region, i.e., the red-framed square in (L). A few donor-derived cells display XX signals, and their nuclei are similar to those of SCC cells in size (white arrow).

transplantation and compatibility with GVHD (Cy5 for the $\mathrm{X}$ chromosome; SpectrumRed for the Y chromosome; Fig. 4). An analysis of normal epithelial cells and carcinoma cells revealed the expression of green $(\mathrm{X})$ signals but not red $(\mathrm{Y})$ signals (Figs. 4 and 5; Tables II and III). Red (Y) signals were detected only in cells with relatively small nuclei compared with those of the epithelial cells, indicating lymphocyte infiltration and compatibility with GVHD (Figs. 4C, 5C and F). 

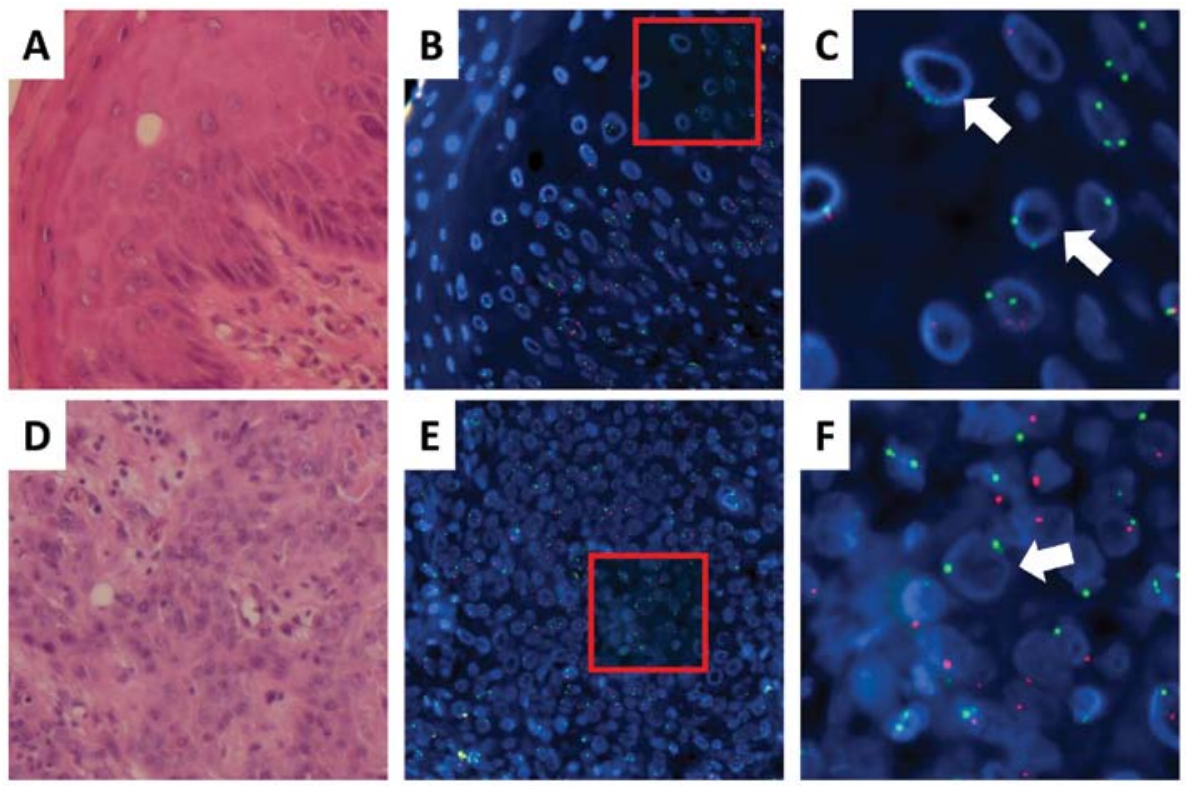

Figure 3. Recipient- and donor-derived oral epithelial cells and carcinoma cells from a male recipient (case 3) of bone marrow stem cells from a female donor. (A) Tissue sections of the normal mucosa were stained with hematoxylin and eosin. (B) Neighboring tissue sections of (A) were examined by FISH to determine the centromeres of the X (Cy5, green) and Y (SpectrumRed, red) chromosomes. (C) A magnified image of the red-framed square in (B). A few donor-derived cells had XX signals and their nuclei were the same size as those of normal epithelial cells (white arrow). (D) Tumor tissue sections were stained with hematoxylin and eosin. (E) Neighboring tissue sections of (D) were examined by FISH to determine the centromeres of the X (Cy5, green) and Y (SpectrumRed, red) chromosomes. (F) A magnified image of the cancer region, i.e., the red-framed square in (E). A few donor-derived cells display XX signals, and their nuclei are similar to those of SCC cells in size (white arrow).
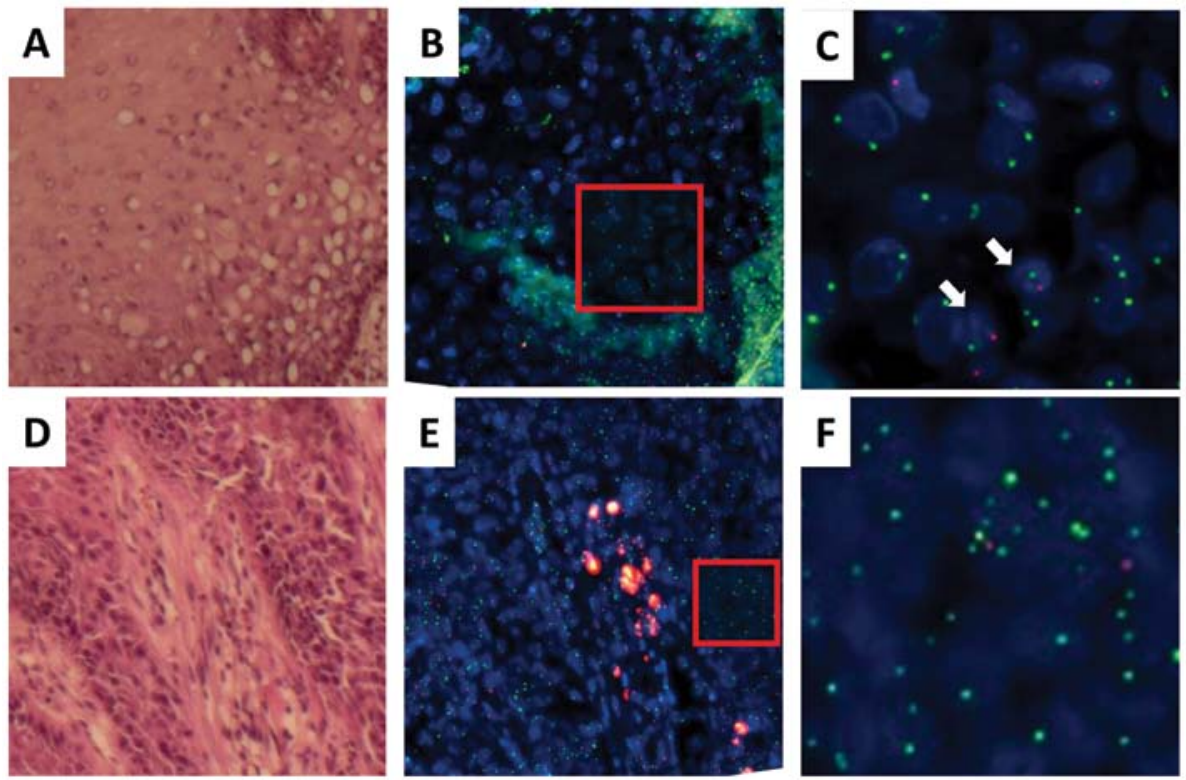

Figure 4. Recipient-derived epithelial cells and carcinoma cells in tongue tissue obtained from a female recipient (case 4-1) of bone marrow stem cells from a male donor. (A) Tissue sections of normal mucosa are stained with hematoxylin and eosin. (B) Neighboring tissue sections of (A) are examined by FISH to determine the centromeres of the X (Cy5, green) and Y (SpectrumRed, red) chromosomes. (C) A magnified image of the red-framed square in (B). A few infiltrating donor-derived cells display Y signals, and their nuclei are smaller than those of normal epithelial cells (white arrow). (D) Tumor tissue sections are stained with hematoxylin and eosin. (E) Neighboring tissue sections of (D) are examined by FISH to determine the centromeres of the X (Cy5, green) and Y (SpectrumRed, red) chromosomes. (F) A magnified image of the cancer region, i.e., the red-framed square in (E).

\section{Discussion}

In this study, we utilized a highly sensitive combination of FISH using gender chromosome-specific probes and histopathological analyses to clearly demonstrate that human epithelial cancer of the head and neck can arise from donor cells after gender-mismatched hematopoietic transplantation (Tables II and III). In a case of esophageal cancer (case 1), almost all cancer cells were donor-derived in the regions examined, whereas a dysplastic lesion was composed exclusively of donor 

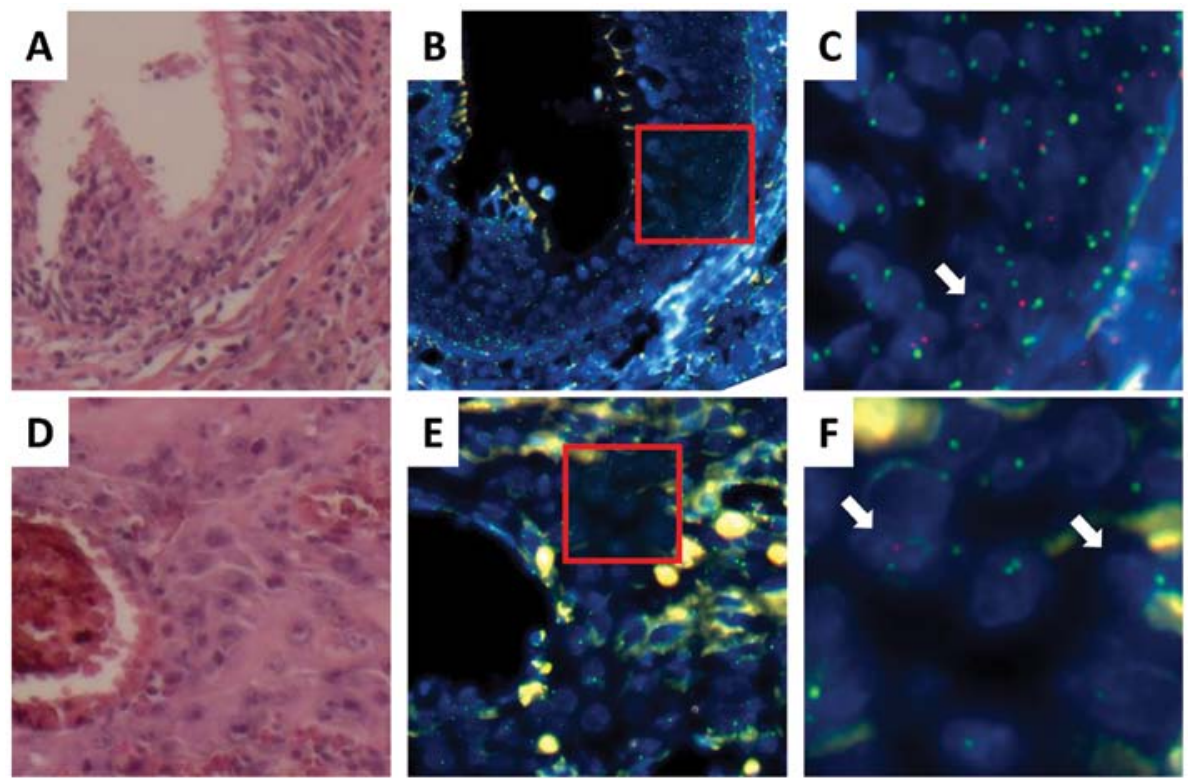

Figure 5. Recipient-derived oral epithelial cells and carcinoma cells from a female recipient (case 4-2) of bone marrow stem cells from a male donor. (A) Tissue sections of normal mucosa are stained with hematoxylin and eosin. (B) Neighboring tissue sections of (A) are examined by FISH to determine the centromeres of the X (Cy5, green) and Y (SpectrumRed, red) chromosomes. (C) A magnified image of the red-framed square in (B). A few infiltrating donor-derived cells display Y signals, and their nuclei are smaller than those of normal epithelial cells (white arrow). (D) Tumor tissue sections are stained with hematoxylin and eosin. (E) Neighboring tissue sections of (D) are examined by FISH to determine the centromeres of the X (Cy5, green) and Y (SpectrumRed, red) chromosomes. (F) A magnified image of the cancer region, i.e., the red-framed square in (E). A few infiltrating donor-derived cells display $Y$ signals, and their nuclei are smaller than those of normal epithelial cells (white arrow).

cells in case 2. Our observations indicated that donor BM contains various cells, including mesenchymal cells, and the collection of hematopoietic cells after HSCT caused those cells to be transferred to the recipient and contributed to the formation of epithelial structures $(7,8)$. Alternatively, some epithelial cell types, even though normal, can free themselves from epithelial structures and migrate into the blood stream through the well-known phenomenon of epithelial-mesenchymal transition, as occurs during the metastasis of solid tumors (10). Although the release frequency of normal epithelial cells from tissues under physiological conditions is uncertain, patients with solid tumors reportedly possess circulating epithelial cancer cells in the peripheral blood and BM (10). In the cases we examined, none of the donors developed late-onset malignancies during the observation period, which reinforced the fact that BM-derived or circulating mesenchymal stem cells, rather than apparent epithelial cells, in donors may change their phenotypes in recipient organs. This notion is compatible with recent studies in which circulating donor-derived BMSCs differentiated into epithelial cells and tumor cells after HSCT in mice and humans (11-30). Nevertheless, a mixture of recipient- and donor-derived origins was involved in cases 2 and 3; therefore, we hypothesized that the initiation and progression of esophageal carcinogenesis may occur in either recipient- or donor-derived single cells in the epithelium and that the adjacent cells may be recruited or accumulated against a background of inflammation and exposure to an immunosuppressant. However, we cannot exclude the possibility of simultaneous transformation in both recipient- and donor-derived cells.

BMSCs are known to differentiate into many types of cells. In the mouse, circulating BMSCs have been reported to differentiate into gastric mucosal cells, lung epithelial cells, renal epithelial cells, keratinocytes, hepatocytes, duct cells, astrocytes and neurons (3-7). In humans, it has been observed that BMSCs differentiate into buccal epithelial cells, keratinocytes, gastrointestinal tract cells, lung epithelial cells, hepatocytes, duct cells, astrocytes and neurons (8-11). In our cases of secondary epithelial cancers subsequent to HSCT, GVHD and exposure to immunosuppressants may have elicited the recruitment and accumulation of BMSC-derived epithelial cells in the tissues (5). The present study suggested the involvement of at least three components in the maintenance and carcinogenesis of head and neck tissues: tissue stem cells in the epithelial layer, circulating stem cells and HSCs.

In mice, BMSCs are reportedly involved in various types of solid tumor formation, including tumors of the epithelium, neural and muscle tissues, fibroblasts and blood vessel endothelium (12,14-25). In human oral SCC, BMSCs have been implicated in mucoepidermoid carcinoma of the parotid glands, invasive ductal carcinoma in the breast, papillary thyroid carcinoma, cervical carcinoma, Kaposi's sarcoma, lung adenocarcinoma, skin SCC, glioblastoma multiforme and pharyngeal SCC (26-31). Although five previous cases suggested donor-derived tumor tissues $(29,31)$, in the present study, we performed highly sensitive FISH using gender chromosome-specific probes and histopathological analyses in cases of head and neck tumors to clearly demonstrate the occurrence of donor-derived human esophageal cancer and dysplasia, a precancerous lesion. This is the first and definite examination to the best of our knowledge. We conclude that BMDCs can contribute to the constitution of epithelial tissues and further the occurrence of carcinogenesis stimulated by chromic inflammation and immunosuppressive conditions. 


\section{Acknowledgements}

This study was supported in part by a Grant-in-Aid for Scientific Research from the Ministry of Education, Culture, Sports, Science and Technology (H.I., M.M.); a Grant-in-Aid from the Third Comprehensive 10-year Strategy for Cancer Control, Ministry of Health, Labor and Welfare (H.I., M.M.); a grant from the Kobayashi Cancer Research Foundation (H.I.); and a grant from the Princess Takamatsu Cancer Research Fund, Japan (H.I.). M.K., Toshihiro Kudo, Daisuke Sakai, Taroh Satoh and H.I. received partial support from Chugai Co. Ltd. and Yakult Honsha Co. Ltd. through institutional endowments.

\section{References}

1. Konno M, Hamabe A, Hasegawa O, et al: Adipose-derived mesenchymal stem cells and regenerative medicine. Dev Growth Differ 55: 309-318, 2013.

2. Puetzer JL, Petitte JN and Loboa EG: Comparative review of growth factors for induction of three-dimensional in vitro chondrogenesis in human mesenchymal stem cells isolated from bone marrow and adipose tissue. Tissue Eng Part B Rev 16: 435-444, 2010.

3. Varon C, Dubus P, Mazurier F, et al: Helicobacter pylori infection recruits bone marrow-derived cells that participate in gastric preneoplasia in mice. Gastroenterology 142: 281-291, 2012.

4. Sung AD and Chao NJ: Concise review: acute graft-versus-host disease: immunobiology, prevention, and treatment. Stem Cells Transl Med 2: 25-32, 2013.

5. Ohtani $\mathrm{N}$ and Hara E: Roles and mechanisms of cellular senescence in regulation of tissue homeostasis. Cancer Sci 104: 525-530, 2013.

6. Du K, Hu Y, Wu K and Huang H: Long-term outcomes of antithymocyte globulin in patients with hematological malignancies undergoing myeloablative allogeneic hematopoietic cell transplantation: a systematic review and meta-analysis. Clin Transplant 27: E91-E100, 2013.

7. Chao YH, Wu HP, Chan CK, et al: Umbilical cord-derived mesenchymal stem cells for hematopoietic stem cell transplantation. J Biomed Biotechnol 2012: 759503, 2012.

8. Bernardo ME, Cometa AM and Locatelli F: Mesenchymal stromal cells: a novel and effective strategy for facilitating engraftment and accelerating hematopoietic recovery after transplantation? Bone Marrow Transplant 47: 323-329, 2012.

9. Majhail NS: Secondary cancers following allogeneic haematopoietic cell transplantation in adults. Br J Haematol 154 301-310, 2010

10. Arwert EN, Hoste E and Watt FM: Epithelial stem cells, wound healing and cancer. Nat Rev Cancer 12: 170-180, 2012.

11. Houghton J, Stoicov C, Nomura S, et al: Gastric cancer originating from bone marrow-derived cells. Science 306 : 1568-1571, 2004.

12. Jenkins RB, Qian J, Lieber MM, et al: Detection of c-myc oncogene amplification and chromosomal anomalies in metastatic prostatic carcinoma by fluorescence in situ hybridization. Cancer Res 57: 524-531, 1997.

13. Krause DS, Theise ND, Collector MI, et al: Multi-organ, multilineage engraftment by a single bone marrow-derived stem cell. Cell 105: 369-377, 2001.
14. Rizvi AZ, Swain JR, Davies PS, et al: Bone marrow-derived cells fuse with normal and transformed intestinal stem cells. Proc Natl Acad Sci USA 103: 6321-6325, 2006.

15. Inokuma D, Abe R, Fujita Y, et al: CTACK/CCL27 accelerates skin regeneration via accumulation of bone marrow-derived keratinocytes. Stem Cells 24: 2810-2816, 2006.

16. Okumura T, Wang SS, Takaishi S, et al: Identification of a bone marrow-derived mesenchymal progenitor cell subset that can contribute to the gastric epithelium. Lab Invest 89: 1410-1422, 2009.

17. Scarlett CJ, Colvin EK, Pinese M, et al: Recruitment and activation of pancreatic stellate cells from the bone marrow in pancreatic cancer: a model of tumor-host interaction. PLoS One 6: e26088, 2011.

18. Matsumoto T, Okamoto R, Yajima T, et al: Increase of bone marrow-derived secretory lineage epithelial cells during regeneration in the human intestine. Gastroenterology 128: 1851-1867, 2005

19. Tran SD, Pillemer SR, Dutra A, et al: Differentiation of human bone marrow-derived cells into buccal epithelial cells in vivo: a molecular analytical study. Lancet 361: 1084-1088, 2003.

20. Mattsson J, Jansson M, Wernerson A, et al: Lung epithelial cells and type II pneumocytes of donor origin after allogeneic hematopoietic stem cell transplantation. Transplantation 78: 154-157, 2004

21. Körbling M, Katz RL, Khanna A, et al: Hepatocytes and epithelial cells of donor origin in recipients of peripheral-blood stem cells. N Engl J Med 346: 738-746, 2002.

22. Miura M, Miura Y, Padilla-Nash HM, et al: Accumulated chromosomal instability in murine bone marrow mesenchymal stem cells leads to malignant transformation. Stem Cells 24: 1095-1103, 2006

23. Li HC, Stoicov C, Rogers AB and Houghton J: Stem cells and cancer: evidence for bone marrow stem cells in epithelial cancers. World J Gastroenterol 12: 363-371, 2006.

24. Liu C, Chen Z, Chen Z, et al: Multiple tumor types may originate from bone marrow-derived cells. Neoplasia 8: 716-724, 2006

25. Guest I, Ilic Z, Ma J, Grant D, et al: Direct and indirect contribution of bone marrow-derived cells to cancer. Int $\mathrm{J}$ Cancer 126: 2308-2318, 2010.

26. Cogle CR, Theise ND, Fu D, et al: Bone marrow contributes to epithelial cancers in mice and humans as developmental mimicry. Stem Cells 25: 1881-1887, 2007.

27. Avital I, Moreira AL, Klimstra DS, et al: Donor-derived human bone marrow cells contribute to solid organ cancers developing after bone marrow transplantation. Stem Cells 25: 2903-2909, 2007.

28. Soldini D, Moreno E, Martin V, et al: BM-derived cells randomly contribute to neoplastic and non-neoplastic epithelial tissues at low rates. Bone Marrow Transplant 42: 749-755, 2008.

29. Janin A, Murata H, Leboeuf C, et al: Donor-derived oral squamous cell carcinoma after allogeneic bone marrow transplantation. Blood 113: 1834-1840, 2009.

30. Hutchinson L, Stenstrom B, Chen D, et al: Human Barrett's adenocarcinoma of the esophagus, associated myofibroblasts, and endothelium can arise from bone marrow-derived cells after allogeneic stem cell transplant. Stem Cells Dev 20: 11-17, 2011.

31. Munakata W, Nomoto J, Takahashi N, et al: Carcinoma of donor origin after allogeneic peripheral blood stem cell transplantation. Am J Surg Pathol 36: 1376-1384, 2012. 